\title{
Poéticas del intruso. Jacques Rancière, lector de Mallarmé*
}

Fecha de recepción: 18 de febrero de 2016

Fecha de aprobación: 29 de junio de 2016

\section{Resumen}

Este artículo analiza el significado de la figura del poeta como "intruso" en la obra de Stéphane Mallarmé, tal como es estudiada por el filósofo y ensayista francés Jacques Rancière en La política de la sirena y La palabra muda. Nuestra intención apunta a mostrar otras definiciones del rol del poeta y del espacio literario en uno de los creadores más destacados del arte contemporáneo.

Palabras clave: Mallarmé, Rancière, poesía francesa, filosofía, literatura comparada.

\begin{abstract}
Alberto Bejarano
Doctor en Filosofía y estética de la Universidad París 8. Docente-Investigador en literatura comparada en el Instituto Caro y Cuervo de Bogotá. Escritor. otrasinquisiciones@hotmail. com.
\end{abstract}

* Artículo de reflexión.
Citar: Bejarano, A. (julio-diciembre de 2016). Poéticas del intruso. Jacques Rancière, lector de Mallarmé. La Palabra, (29), 129-137. doi: http://dx.doi. org/10.19053/01218530.n29.2016.5706 


\section{la palabra}

Poetics of the Intruder. Jacques Rancière's

Mallarmé

\section{Abstract}

This essay analyzes the meaning of the figure of the poet as intruder in Stéphane Mallarmé's works, as it is studied by the French critical theorist and philosopher Jacques Rancière in Politics of the Siren and Mute speech. Our intention is to reflect about and expand definitions of the role of the poet and the literary space in one of the most remarkable creators of contemporary art.

Keywords: Mallarmé, Rancière, French poetry, philosophy, comparative literature.

\section{Poétiques de l’intrus. Jacques Rancière, lecteur de Mallarmé.}

\section{Résumé}

Cet article analyse la figure du poète comme « intrus » dans l'œuvre de Stéphane Mallarmé, telle qu'elle est étudiée par le philosophe et essayiste français Jacques Rancière dans « La politique de la sirène » et «La parole muette ». Nous voulons montrer autres définitions du rôle du poète et de l'espace littéraire chez un des créateurs le plus importants de l'art contemporain.

Mots-clés : Mallarmé, Rancière, poésie française, philosophie, littérature comparée. 
"Prends ce sac, Mendiant! Tu ne le cajolas

Séniles nourrisson d'une tétine avare

Afin de pièce à pièce en égoutter ton glas.

Tire du métal cher quelque péché bizarre

Et vaste comme nous, les poings pleins, les baisons

Souffles-y qu'il se torde! Une ardente fanfare"

Mallarmé, Aumône

[“ $¡$ Toma el saco, Mendigo! No lo has engatusado

cría senil de avaro pezón, para moneda

a moneda agotar tu toque de agonía.

Del caro metal saca algún pecado extraño

$\mathrm{Y}$ vasto cual nosotros, a manos llenas, lo besamos

¡sopla que se refuerza! una ardiente fanfarria...”]

Mallarmé, "Limosna" (p. 60)

\section{Introducción}

Entendemos la literatura comparada no como una mera división de la teoría literaria ni como un simple juego de influencias más o menos lineales entre autores, sino como una reconfiguración permanente de todo tipo de interacciones posibles entre obras de distinto origen y tiempo. Este es el caso del presente artículo, en el que se estudia el diálogo transversal entre Mallarmé y su recepción en la filosofía francesa contemporánea. Se trata de explorar los espacios abiertos para re-leer los clásicos, siguiendo la línea de autores como Fusillo (2012): "El lector de las obras posmodernas está llamado a desafíos cada vez más difíciles, ya sea por recorridos simultáneos a través de géneros, lenguajes y narraciones multidimensionales, ya sea por los espacios enigmáticos que la literatura deja abiertos" (p. 188).

Partiremos de la definición de estética de Rancière no como teoría del arte, sino como régimen de lo sensible, como modo de visibilidad y decibilidad de los cuerpos donde se inserta una noción de literatura que reconfigura necesariamente lo que Blanchot llamaba ya "espacio literario", problematizando los puntos de partida sartreanos (para qué se escribe, para quién se escribe) y bartheanos (qué es un texto, qué es un autor, qué es un lector), con el fin de evidenciar una vuelta de tuerca de 1) la estética; 2) la literatura; y, 3) la literatura comparada. Para Rancière (2009), "El arte y la política comienzan cuando se perturba ese juego común en que las palabras se deslizan continuamente bajo las cosas y las cosas bajo las palabras. Comienzan cuando las palabras se hacen figuras, cuando llegan a ser realidades sólidas, visibles" (p. 83).

Se trata de una larga historia que viene desde Walter Benjamín y pasa por Adorno, Sartre, Blanchot, Deleuze, Rancière, Derrida, Badiou, Lefort, Abensour, y entre nosotros por autores como Rafael Gutiérrez Girardot, para citar solo algunos ejemplos. Todo esto nos lleva a querer investigar sobre la potencia de la escritura y sus metamorfosis en la literatura contemporánea, comenzando por el caso de Mallarmé. Se trata también de un ejercicio en una doble vía: de los filósofos hacia los escritores y de los "pensadores literarios" (Borges) hacia los filósofos, 
más allá o más acá de (in)ciertas fronteras.

\section{Repensando los clásicos}

Nuestro propósito es reflexionar sobre la contribución filosófica de Jacques Rancière a los estudios estéticos en los últimos diez años. De esta forma, si bien nos apoyaremos en las obras monográficas de Rancière sobre Mallarmé, también nos apoyaremos en su obra a partir de la publicación de "La división de lo sensible" en el año 2000 hasta la aparición de "et tant pis pour le gens fatigués” en 2009. Buscamos insistir en un panorama crítico de la obra (en curso) de Rancière en torno a conceptos como "emancipación", “división de lo sensible", o "crítica a la modernidad", entre otros.

A lo largo de las dos últimas décadas, el filósofo francés Jacques Rancière se ha dedicado a repensar el lugar y el sentido múltiple de la literatura con respecto a su historia $\mathrm{y}$ a su relación con la filosofía. En ese camino, la poesía y la prosa de Stéphane Mallarmé ha ocupado un lugar central en sus reflexiones, hasta el punto de dedicarle el único libro monográfico que ha escrito (Politicas de la sirena, 1996) y ser el epicentro de diversas discusiones con el Mallarmé de Jean Paul Sartre, Maurice Blanchot, Jacques Derrida y, en especial, con el de Alain Badiou. En este texto, indagaremos por la lectura filosófica que lleva a cabo Rancière del poeta simbolista Mallarmé y por las implicaciones de su poética en su propia filosofía.

La presencia del poeta francés Stéphane Mallarmé en la filosofía francesa contemporánea puede situarse a partir del texto de Jean Paul Sartre, Mallarmé, la lucidez y su cara de sombra, cuya primera versión se publica después de Qué es la literatura (1946); allí, Sartre define al poeta simbolista como el arquetipo del artista comprometido. Se trata, a primera vista, de algo paradójico en la medida en que Mallarmé siempre fue visto y tratado como un poeta "apolítico" e incluso "reaccionario" (por su "no-intervención" en los principales acontecimientos políticos de su época: la Comuna de París de 1871 y el Affaire Dreyfus durante la III República francesa en las décadas de 1880 y 1890) y fue representado durante décadas como una especie de espectro encerrado en su Torre de Marfil de la Calle de Roma en París, tal como fue descrito por Karl Huysmans en su novela À Rebours. Por otra parte, a través de la figura de Mallarmé, Sartre problematizará su propio concepto de "compromiso", al precisar que el compromiso del artista solo tiene lugar en el espacio de la Obra (de allí proviene su fascinación por autores como Charles Baudelaire, Gustave Flaubert o Jean Genet, escritores que se distan- cian del lugar-común del artista-comprometido).

Sin embargo, Sartre ligará el destino de Mallarmé a la revolución de 1848 y verá en el poeta francés el primer poeta completamente "laico". Ya en 1955, Maurice Blanchot iniciaba también su ensayo "La experiencia de Mallarmé" haciendo alusión a la relación entre los "dioses idos" y los "nuevos poetas", según Mallarmé: "desafortunadamente, cavando (en) el verso a tal punto, me encontré con dos abismos que me desesperan. Uno es la Nada [...]" (Blanchot, 1992, p. 36), y agrega Blanchot: "la ausencia de Dios, el otro es su propia muerte" (p. 37). Blanchot ve en Mallarmé el "poeta-filósofo" que parte y llega al Silencio y que crea una/otra lengua-del-silencio, distante de la lengua ordinaria y de la lengua del pensamiento. Para Blanchot (1992), "el silencio es el oficio y la palabra de la significación" (p. 41); y continúa diciendo: "a partir de ahora, no es Mallarmé quien habla, sino el lenguaje se habla, el lenguaje como obra y la obra del lenguaje" (p. 42).

Para Blanchot, la experiencia de Mallarmé tiene que ver con el origen y la desaparición de la Obra y de la literatura como único "fin" posible de su relación ontológica con el mundo. La Obra es el espacio mismo del "final" y de la "muerte" para Blanchot, un tema que lo acompañará a lo largo de sus 
búsquedas y que bifurcará ciertos caminos con/en Jacques Derrida y Jean Luc Nancy, por ejemplo. Hacia 1972, Derrida publica a su vez un largo ensayo sobre Mallarmé, titulado "La doble función", publicado en su libro La diseminación. En Derrida (2008), también la literatura muere en el momento de su nacimiento... "la historia de Platón y Mallarmé fue también una historia de la literatura, si se admite que la literatura nació allí y murió, su acto de nacimiento como tal, la declaración de su nombre, coincidió con su desaparición" (p. 225).

Para Blanchot (1992), el trabajo de la obra consiste, en buena medida, en activar nuestra "mirada doméstica del espectador..." (p. 51). Frente a esa idea, Jacques Rancière opondrá su tesis del espectador emancipado. A diferencia de Blanchot, Rancière verá en Mallarme el poeta-de-los-filósofos que devela las posibilidades creativas inesperadas de La palabra muda, entendida como un espacio emancipador en el que una "nueva" idea de Libro abre las puertas al Mundo del Lector de la nueva era democrática en la que la palabra es cada vez-más-viva, lo que justamente desembocará en las vanguardias de principios del siglo XX. En ese sentido, la presencia de Mallarmé a lo largo del siglo XX se puede rastrear no solamente en la poesía, sino en las artes plásticas y visuales, en los experimentos, montajes, composiciones y ensamblajes de artistas tan diversos como Man Ray o Francis Bacon.

Por su parte, Alain Badiou publica en 1998 un capítulo sobre Mallarmé en su Pequeño manual de inestética. ${ }^{1}$ Para él, Mallarmé es la expresión ambigua del Maestro que hace "surgir" las Verdades en medio del vacío y de su propia desaparición: "la Verdad surge, como un golpe de dados ideal inscrito en el cielo nocturno [...] hay que recomponer para nuestro tiempo un pensamiento de la verdad que sea articulado sobre el vacío sin pasar por la figura del maestro [...] una verdad comienza por un poema del vacío[...]" (Badiou, 2008 , pp. $80,86,89$ ).

\section{Mallarmé y la filosofía}

Después de estas sucesivas lecturas filosóficas de Mallarmé, de Sartre a Badiou, pasando por Blanchot y Derrida -que resumimos aquí de manera extremadamente sucinta-, Rancière escribirá dos ensayos sobre Mallarmé en donde profundi- zará su propia lectura del poeta, iniciada en 1996 con su libro, Mallarmé. La politique de la sirène. Dichos ensayos son, "El intruso. La política de Mallarmé" y "El poeta en el filósofo. Mallarmé en Badiou", publicados en Política de la literatura (2007). Por razones de tiempo, nuestro análisis se concentrará principalmente en el ensayo, "El intruso. La política de Mallarmé".2

Pero, volviendo a nuestro punto de partida, aclaremos que la idea que Sartre tiene de "política" y de sus relaciones con el arte entran en un plano diferente a una simple "participación" del autor en un "affaire (asunto) político". Rancière partirá de una idea similar a la hora de proyectar su propia visión de Mallarmé, como lo veremos más adelante. A su vez, para la filosofía francesa, Mallarmé se ha convertido en todo un "affai$r e$ " en el que el poeta deviene un campo-de-combate a la hora de definir qué entiende cada pensador por arte y política. De esta forma, podemos decir que la expresión "affaire" da cuenta de la compleja relación entre lo estético y lo político en sus múltiples resonancias. Para Sartre, el artista debe comprometerse con su tiempo dentro de la obra

\footnotetext{
Así como Derrida incursiona en Mallarmé en una mirada doble con el Crátilo de Platón, Badiou experimenta en literatura comparada con una lectura cruzada de Mallarmé y el poeta pre-islámico Ben Rabi'.

2 Ver también otros textos de Rancière donde se refiere a Mallarmé: L'inesthétique d'Alain Badiou: les torsions du modernisme; Rimbaud, les voix et le corps y La escritura de la idea en el libro La palabra muda. Para profundizar en la lectura de Badiou sobre Mallarmé, ver también sus libros El ser y el acontecimiento y Condiciones.
} 
de una manera total; Mallarmé lo apasiona porque ve en él el máximo representante de una búsqueda estética total: la búsqueda del Libro perfecto, el Libro que, inevitablemente, como lo mencionará Blanchot, siempre es el Libro-por-venir ${ }^{3}$. Pero, ¿cómo lee Sartre a Mallarmé?, $\mathrm{y}$, ¿qué tipos de textos del poeta francés privilegia en su análisis? Estas preguntas son esenciales a la hora de comprender la originalidad y especificidad de la lectura de Rancière con respecto a la reflexión sartriana. Para decirlo en otros términos, me gustaría sugerir como primera hipótesis de lectura que, Mallarmé es una de las claves de interpretación para estudiar las "políticas de la literatura" en Rancière -y para llevar a cabo además un estudio comparado con Badiou, Derrida y Blanchot, por ejemplo, quienes también le dedican memorables textos al poeta francés- no solo porque ha sido el único autor al que le ha dedicado una monografía, sino porque su nombre es usado como escenario de confrontación hospitalaria -la hos(pi) ti(a)lidad derridiana- con otros filósofos. A diferencia de Sartre, Mallarmé es para Rancière más que un "artista-comprometido", es el primer poeta-de-la-era-democrática y paradigma posible de una política y poética plu- rales, tal como son enunciadas por Rancière en otros textos. Mallarmé no es singular, dirá Rancière, solo por sus ideas con respecto a la literatura y a la sociedad, sino por la forma como introduce transformaciones revolucionarias en la manera de escribir y leer un texto, en la indistinción entre géneros, y en la puesta en escena de la Vida como ejercicio experimental inagotable. La mirada política sobre/desde Mallarmé no se reduce a estudiar las ideas políticas del autor, sino a poner a resonar sus propuestas estéticas más allá de su tiempo, para generar nuevas configuraciones de lo sensible que sugieran otras formas de vida.

De esta manera, una de las críticas que Rancière le hará a Sartre se dirige a problematizar la forma como Sartre separa la poesía de la prosa como dos tentativas irreconciliables. Derrida (2008) también había profundizado en esa idea desde la Gramatología, y lo hará aún más en La doble función: «la práctica versificadora se confunde con la literatura que "sobrepasa el género" y desborda en sus efectos como en sus principios, la oposición vulgar de la prosa a la poesía» (p. 339). ${ }^{4}$
Para Rancière, incluso antes de Mallarmé, ya Baudelaire había roto esas fronteras con sus poemas en prosa del Spleen de París. Además, Mallarmé evidencia no solo la fragilidad de una rígida idea de la literatura ligada a la defensa de los géneros y los cánones, sino que radicaliza una idea de literatura unida a lo burgués como expresión de una pura artificialidad y al amusement (divertimento) asociado al ocio y a la auto-celebración como clase triunfante, ya que al dedicarse a asumir la "crisis del verso" de finales del siglo XIX (no solo en cuanto forma), Mallarmé corroe las instituciones que soportan supuestamente lo literario, mostrando la inexpugnabilidad del hecho estético en toda su potencia de enunciación y misterio.

En su ensayo "El intruso. Política de Mallarmé", publicado en Política de la literatura, Rancière (2009) comienza con una cita del libro de Sartre sobre Mallarmé que hemos evocado desde el principio...

hasta entonces el Verbo era el intermediario entre el poeta y el lector; ahora es una columna de silencio que florece solitaria en un jardín escondido: si el lector escala los muros, si

3 Ver también las múltiples lecturas, de ida y vuelta, de Derrida hacia Blanchot, en especial su texto, El libro por venir, publicado en Papel-Máquina.

4 Derrida y Rancière comparten (partagent) también la escena/la sesión de una relectura del Crátilo de Platón desde la mirada de Mallarmé en torno al concepto de mimesis. 
ve chorros de agua, flores y mujeres desnudas, debe sentir primero que todo esto no le pertenece, ni ha sido reunido para él (p. 93).

Ahora bien, para Rancière todo pasa por la "fundación" del Verbo en Mallarmé destinado a ser algo diferente a la petrificación blanchotiana/derridiana y a la elitización que reprocha Sartre, pues sugiere Rancière (2009): "el Verbo (de Mallarmé) instituye una morada nueva de la comunidad" (p. 94). Mallarmé es el primer poeta democrático $\mathrm{y}$ no un decadente, dice Rancière, porque asume la tarea de escribir para/por el "género humano en su totalidad" (p. 94); y la famosa "crisis del verso" en Mallarmé tiene una línea subterránea de continuidad con el siglo de las revoluciones y con el lugar "nuevo" del hombre en el mundo. "Se trata de redefinir lo que hace una Comunidad humana más de un simple sistema de intercambios" (Rancière, 2009, p. 95). Es en este punto donde surge y puede apreciarse mejor qué entiende Rancière por "política de la literatura", es decir "cuando el acto insensato del poeta encuentra la división de la palabra como modo que instituye una cierta comunidad... la escritura es un gesto material insensato que dispone del sentido, que lo transforma en maneras de hacer y de ser, y que inscribe así el carácter de una comunidad" (p. 96). A Ran- cière le interesa definir en otros términos el espacio-del-poema y su relación con el espacio del pueblo, de la comunidad, y es en esa operación filosófica donde Mallarmé ocupa un lugar esencial en su obra, ya que el poeta no es visto a través del prisma del vidente, el maestro o el genio, sino como un "intruso", como un ser ambiguo que desata de manera "insensata" (la expresión más recurrente en Rancière) una nueva forma de pensar, ser y hacer comunidad, a través del arte como espacio de lo común y lo inesperado. Es por ello que para Rancière ni el Silencio ni la Muerte son el destino de la obra, y cuando utiliza la expresión "la palabra muda" se refiere más bien a una confrontación entre formas de lo decible y lo visible que nunca son acalladas del todo. "El intruso es aquel que cambia de lugar y de identidad" (Rancière, 2010, p. 98). Mallarmé no es el poeta-esteticista y estetizante que nos ha entregado la tradición literaria, sino la figura del intruso, del lector de "fait divers" (crónicas rojas) de su época que convive con las explosiones revolucionarias $\mathrm{y}$ con los cambios profundos en la forma como se mueven y se "ubican" los cuerpos en la vida social. Rancière lee a Mallarmé desde una clave completamente distinta a Sartre, Blanchot, Derrida y Badiou: lo lee excavando por igual en sus sonetos parnasianos, en sus traducciones y crónicas, y en especial en sus "chansons bas" (canciones bajas), historias de remendones, vendedoras de hierbas aromáticas, peones camioneros, vendedores de ajos y cebollas, cristaleros, vendedores de periódicos, ropavejeros y mujeres de obreros, que por lo general han sido relegadas o subestimadas a la hora de acercarse a Mallarmé. Veamos, por ejemplo, su poema "La Ropavejera":

\section{"La Marchande d'habits.}

Le vif dont tu regardes Jusques à leur contenu Me sépare de mes hardes

\section{Et comme un dieu je vais nu"}

["La agudeza con que miras incluso su contenido me separa de mis trapos y como un dios voy desnudo".] (Mallarmé, 2008, p. 151).

Lo leemos en francés para remarcar dos puntos: el sentido de "vif" y el juego fonético en francés del verso "je vais nu" que significa "voy desnudo", pero a la vez sonaría como "he venido"... El intruso es aquel que viene sin ser esperado $\mathrm{y}$ perturba el orden de los cuerpos y de los lugares. El poeta como intruso, Mallarmé como arquetipo del intruso en la modernidad y en la era de la democracia resume, en buena medida, la idea de política y de estética en Ranciére. El poeta no es quien habla por los otros y le da voz a los que supuestamente no tienen voz. El poeta no es el que anuncia una Verdad, por muy 
plural que esta sea. El Poeta es el intruso inagotable, es el testigo no llamado que interrumpe el orden ya configurado y nos señala otras miradas posibles, muchas veces impensadas. He ahí el punto de tensión entre literatura y filosofía.

Por otra parte, Rancière (2010) estudia una serie de textos menores de Mallarmé (en el sentido de lo menor en Deleuze, como una resistencia), como "Un espectáculo interrumpido" y "Conflictos", y propone esta definición del poeta:

[...] el deber del poeta no consiste solamente en "comprender" el misterio sino en constituirlo... el misterio poético constituido por la masa de trabajadores cortando el horizonte se convierte en el emblema de ese deber. Para consagrarlo, hay que glorificar aquello que se disimula a la gloria, transformar un anonimato eterno en eternidad de lo anónimo, en gloria de lo cualquiera y de cualquiera. (p. 101).

En eso consiste la transformación de los roles en la era democrática, en invertir el orden de la "eternidad": el poeta como intruso modifica la división de lo sensible, y en un mundo sin dioses se entrega ya no a la disolución inevitable del ser en el vacío de Blanchot o Derrida, sino a la emulación y recreación de lo anónimo y ultra-terrenal. Lejos de cualquier nostalgia del "tiempo de los poetas" (Badiou, 2008), Rancière (2009) ve en el poeta, y en especial en Mallarmé, aquel que (con su intrusión)

se convierte en un personaje indispensable para aquel que parece venir indiscretamente a visitar y a ofender con su ocio de paseante... la intrusión del paseante rompe la cadena de los intercambios y del trabajo reproductor (del capital) ... el intruso es aquel que tiene la vocación comunitaria e igualitaria suprema. (p. 102).

Para Rancière, Mallarmé es por estas razones, el poeta del "Conflicto" no solamente entre clases sociales (aristocracia y democracia, según las propias palabras del poeta) sino entre formas de ver y decir la literatura con respecto a la política. Según Rancière, Mallarmé está en una situación paradójica con respecto a las posibilidades de apertura de la palabra y sus límites; y, por eso, la figura del intruso no es para él una especie de manifiesto o arquetipo del lugar del artista en la sociedad. Se podrían establecer puentes, conexiones interesantes con miradas contemporáneas como las que sugiere Borrero (2012): “el lenguaje entonces nos da la pista del funcionamiento, -tensión y complementariedad-, de los planos que constituyen el ser, el del significado proposicional, que se asocia con lo simbólico, representativo...y otro registro no lingüístico, misterioso...que opera a través de la sensibilidad" (p. 56).

De hecho, no hay en Rancière una pretensión de "elevar" al artista a la cabeza de un pueblopor-venir en la medida en que su idea de democracia e igualdad pasan necesariamente por un constante cambio de lugares y posiciones donde la potencia del John Doe, del Juan Nadie (por el film de Frank Capra), del cualquiera, no puede ser abolida ni siquiera por un golpe de dados...

\section{Conclusión}

La lectura filosófica que propone Rancière de la obra completa de Mallarmé (incluyendo poemas en prosa, cartas, poemas bajos, etc.), nos permite revisitar su obra desde otras perspectivas que, en parte, prologan la tradición crítica fiel a los filósofos franceses de la segunda mitad del siglo XX (desde Sartre), considerando al poeta del golpe de dados, como el más filosófico de los poetas franceses. Ahora bien, la singularidad de la reflexión de Rancière consiste en definirlo bajo la figura del "intruso", como aquel que irrumpe para perturbar el orden de lo sensible, a través de sus juegos con la palabra y el espacio de la página en blanco. De 
allí la definición que nos brinda Rancière de Mallarmé, como el primer poeta de la era democrática. De esta manera, podemos comprender mejor por qué Mallarmé no es un "poeta para poetas" ni un esteticista encerrado en su torre de Babel, sino, por el contrario, un revolucionario de la forma y de la expresión poética que cuestiona los márgenes y los confines de lo decible y lo visible.
Este es el singular y novedoso aporte que nos sugiere Rancière, una muestra muy sugestiva sobre las potencias de las lecturas transdisciplinares que no se limitan a continuar una habitual historia de la literatura hecha de clasificaciones y etiquetas más o menos fijas que, por lo general, esconden o invisibilizan la potencia constitutiva de autores como Mallarmé que irrumpen en el espacio literario con dife- rentes efectos que sobrepasan la literatura misma. En la medida en que se profundicen este tipo de diálogos entre filosofía y literatura, podremos progresivamente crear nuevas lecturas sobre los clásicos en un sentido inactual, es decir, buscando siempre lo que pueden decirnos no solamente de su tiempo, sino en especial, sobre el nuestro.

\section{Referencias}

Blanchot, M. (1992). El espacio literario. Barcelona: Paidós.

Badiou, A. (2008). Pequeño manual de inestética. Buenos Aires: Prometeo.

Borrero, J. (2012). «Muchacha no vayas al bosque»: orientaciones para una literatura en el campo expandido. La Palabra (20), 49-67. Recuperado de http://revistas.uptc.edu.co/revistas/index.php/ la_palabra/article/view/958/958

Derrida, J. (2007). La diseminación. Madrid: Fundamentos.

Fusillo, M. (2012). Estética de la literatura. Madrid: La balsa de la medusa.

Mallarmé, S. (1945). Oeuvres complètes. París: Bibliothèque de la Pléiade.

Mallarmé, S. (2008). Poesías seguidas de una tirada de dados. Madrid: Ediciones Hiperión.

Rancière, J. (2009). La palabra muda. Buenos Aires: Eterna cadencia.

Rancière, J. (2010). Política de la literatura. Buenos Aires: Libros del Zorzal.

Rancière, J. (1996). Mallarmé. La politique de la sirène. París: Hachette.

Sartre, J.P. (2007). ¿Qué es la literatura? Buenos Aires: Losada.

Sartre, J.P. (2009). Mallarmé la lucidez y su cara de sombra. Madrid: Arena Libros. 